\title{
The Artificial Immune System Approach for Smart Air-Conditioning Control
}

\author{
Zenon Chaczko, Shahrzad Aslanzadeh, and Jonathan A. Kuleff
}

\begin{abstract}
Biologically inspired computing that looks to nature and biology for inspiration is a revolutionary change to our thinking about solving complex computational problems. It looks into nature and biology for inspiration rather than conventional approaches. The Human Immune System with its complex structure and the capability of performing pattern recognition, self-learning, immune-memory, generation of diversity, noise tolerance, variability, distributed detection and optimisation - is one area that has been of strong interest and inspiration for the last decade. An air conditioning system is one example where immune principles can be applied. This paper describes new computational technique called Artificial Immune System that is based on immune principles and refined for solving engineering problems. The presented system solution applies AIS algorithms to monitor environmental variables in order to determine how best to reach the desired temperature, learn usage patterns and predict usage needs. The aim of this paper is to explore the AISbased artificial intelligence approach and its impact on energy efficiency. It will examine, if AIS algorithms can be integrated within a Smart Air Conditioning System as well as analyse the impact of such a solution.
\end{abstract}

Keywords-artificial immune system, biologically inspired computing, evolutionary computation.

\section{INTRODUCTION}

$\mathbf{T}$ HE Australian Bureau of Statistics states that $11 \%$ of total energy used in Australia is by Households [1]. It also states that in 2005 alone, $78 \%$ of all households had some form of room heating. Room heating is seen as a major contributor to household energy bills, accounting for 39\% of total household energy use and $14 \%$ of the residential sector greenhouse gas emissions. The objective of this paper is to develop an understanding of the relevant biologically inspired computing paradigms that may be used to aid the development of the smart air conditioning system. The above mentioned objective fits in well with the growing trends for a "Smart Home" solution, while also addressing the energy and environmental concerns that plague the concept. Additionally, it contributes to the current research into viable areas to which AIS algorithms can be applied.

\section{A. Traditional Air Conditioning System}

Fundamentally, an Air Conditioner (A/C) can be viewed as a refrigerator without the insulated box.

The AC unit uses the evaporation of a refrigerant, like Freon, to provide cooling. The mechanics of the Freon evaporation

Z. Chaczko and S. Aslanzadeh are with the Faculty of Engineering and IT, University of Technology, Sydney, Bld 1 Level 22 Broadway, Ultimo 2007, NSW, Australia (e-mails: zenon.chaczko@uts.edu.au, shahrzad.aslanzadeh@uts.edu.au).

J. A. Kuleff is graduate of University of Technology, Sydney, NSW, Australia (e-mail: jonathankuleff@gmail.com). cycle are the same in a refrigerator as in an air conditioner. Seen in the figure above the Freon liquid runs through an expansion valve, and in the process it evaporates to become cold, low-pressure Freon gas (seen as light blue). This cold gas runs through a set of coils that allow the gas to absorb heat and cool down the air inside the building. The compressor compresses cool Freon gas, causing it to become hot, highpressure Freon gas (seen on the left in Fig. 1). This hot gas runs through a set of coils so it can dissipate its heat, and it condenses into a liquid. This is the basic concept behind air conditioning.

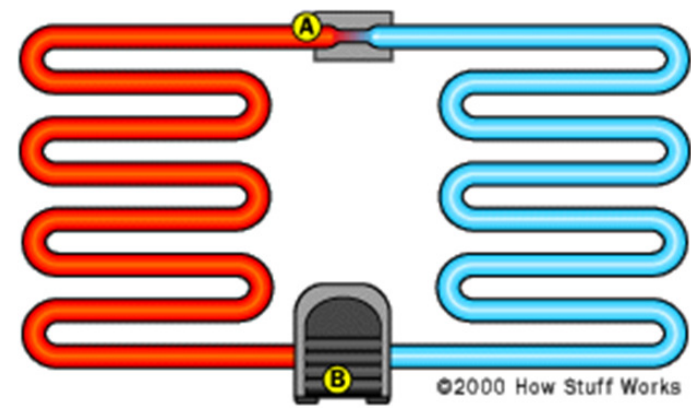

(A) Expansion valve

(B) Compressor

\section{Diagram of a typical air conditioner}

Fig. 1. The evaporation cycle in an air conditioner.

Selecting an air condition system for a room or a home is not an easy task, due to the complexity of thermal dynamics, room features such as its size, the height of the ceiling, amount of windows, amount of internal walls, how many users are generally in the room and many other features can affect the cooling or heating capacity required. One might wrongly assume that buying a unit with a higher heating or cooling capacity is better, as these units would cycle faster making them less effective while wasting energy.

Others might assume that buying a unit with a lower heating or cooling capacity would not make a great difference, again they would be wrong. These units would be on all the time working at its peak output reducing the lifetime of the appliance faster than normal and again using excessive amounts of energy. It would then seem that not all air conditioners are created equal and the Goldilocks Syndrome applies, not too small, not too large but just right. Scientists around the world are working on research to calculate the 'just right' amount needed to cool or heat a room, so for the purpose of this paper we will not look into this further. 
Usage patterns of users are seen as a major contributor to household energy wastage. A common misconception held is that by increasing/decreasing the set temperature on the unit, it will work faster. However, this is incorrect, as units have a fixed capacity output which means that they would only work harder and for longer. Other user patterns for energy wastage include leaving a room for long periods of time and leaving a unit running while they sleep.

\section{B. Energy Efficiency}

In our current global climate and ever increasing trend to 'go green', energy efficiency is increasingly on the mind of consumers. Governments around the world have created systems for comparing the energy efficiency of household appliances. The U.S. Environmental Protection Agency and the U.S. Department of Energy have estimated with the help of the ENERGY STAR program, that in 2007 alone the U.S.A. avoided greenhouse gas emission equivalent to those from 27 million cars, an estimated saving of $\$ 16$ billion in utility costs. In Australia air conditioning units are one of many appliances that are regulated for energy efficiency in the Energy Label system. Figure 2 depicts an example of the label found on a reverse cycle air conditioner. The label allows consumers to compare units at a glance. The more stars it has, the more energy efficient the unit is. It is estimated that hundreds of dollars can be saved over the lifetime of a unit by only selecting a more efficient unit, even though they are generally much more expensive. The star rating for air conditioners is determined by the Energy Efficiency Ratio (EER) tests for cooling and the Coefficient of Performance (COP) for heating. The EER and COP are defined as:

EER = rated cooling capacity output $(k W) /$ cooling input power $(\boldsymbol{k W})$

COP = rated heating capacity output $(k W) / h e a t i n g$ input power $(k W)$

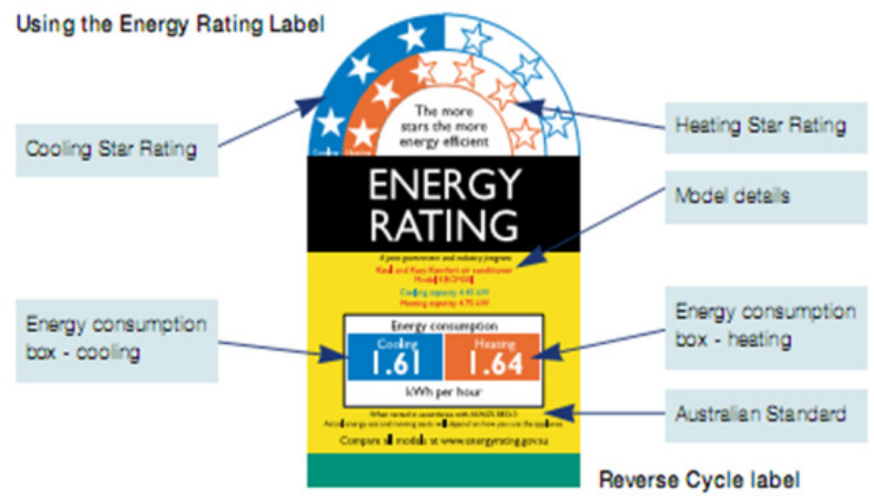

Fig. 2. Example of the energy rating label.

Typically, the EER and COP are in the range 2.0 to 3.5 (meaning that the cooling or heating output is 2 to 3.5 times greater than the power input and with the efficiency between $200 \%$ to $350 \%$ ).

\section{ARtificial IMmUne System}

\section{A. The Human Immune System (HIS)}

Traditional methods used to create systems with artificial intelligence currently have many issues and not always are able to produce the desired results. Over the years, biological inspired alternatives have emerged; one of the more recent paradigms is the Artificial Immune System (AIS), inspired by the principles and processes of the Human Immune System (HIS). The concept of AIS has been developing and gaining momentum since the mid 90's in solving computational problems from mathematics, engineering, and information technology. In computer science terms, and compared to other biological inspired paradigms, AIS is still in its infancy with much still to be discovered, however showing some good prospects [2].

Understanding how HIS works is an important step towards developing an artificial immune system and determining its application areas. The basic HIS concepts will be presented in brief but relevant to the purpose of this paper.

\section{B. The Immune System}

Immunity is defined as "resistance to disease, specifically infectious disease". The immune system is a collection of cells, tissues and molecules that help resist infections. Their coordinated reaction to infectious microbes is the immune response. The HIS is a complex defensive network, comprising of multiple levels, each employs different techniques for defense. At a non-cellular level, the first line of defense is provided by skin; a physical barrier to pathogens. Chemically, skin $\mathrm{pH}^{17}$ levels are maintained below seven (acidic), which inhibits bacterial growth. The Second technique is the use of biochemical barriers such as fluids (tears, perspiration), enzymes (saliva, stomach chemicals) and mucous (nasal secretions) produced by the body. Biochemical substances are combined with mechanical actions such as sensing, coughing and emesis (vomiting) to eject objects from the body [3]. It can be said that the physiologic function of the immune system is to prevent infections and to eradicate established infections. If the intruder bypasses the previous defenses, the HIS deals with it on a cellular level initially with the innate immune system. If the intruder still poses a threat the adaptive immune system represents the last line of defense. My focus will be directed towards the innate and adaptive immune and in the following sections I shall look at its properties and mechanisms.

1) Innate and Adaptive Immune System: The term innate immunity, refers to the fact that this type of host defense mechanism is always present in healthy individuals, prepared to block the entry of microbes and to rapidly eliminate microbes that do succeed in entering host tissues. It provides a nonspecific defensive barrier to pathogens, i.e. all pathogens are attacked equally. The innate immune system remains the same throughout our lifetime and does not change with experience or age, it is inherited from our parents and is genetically based. Although, the innate immunity can effectively combat many infections, microbes pathogenic for humans (i.e. capable of causing disease) have evolved to resist it. Defense against 
these infectious microbes is the task of the adaptive immune response.

Adaptive immunity is a form of host defense mechanism that is stimulated by microbes that invade tissues, that is, it adapts to the presence of microbial invaders. Whereas, the mechanism of innate immunity recognizes structures shared by classes of microbes, the cells of adaptive immunity, namely lymphocytes, express receptors that specifically recognize different substances produces by microbes as well as noninfectious molecules. These substances are called antigens [4]. However, there are many millions of other lymphocytes each carrying different types of receptors and does not limit response to different antigens. Upon biding with an antigen, the lymphocyte is activated to divide and produce clones. The clones secrete antibodies which contain the preceptors that will bind to the original antigen that spawned the cloning process. This process of cloning ensures that a large number of lymphocytes with a specific response are created puts the maturation of lymphocytes process into perspective.

There are two types of adaptive immunity, called humeral immunity and cell-mediated immunity that are mediated by different cells and molecules and are designed to provide defense against extracellular microbes and intracellular microbes, respectively.

Proteins called antibodies, which are produces by cells called B-lymphocytes, mediate humeral immunity. One of the more important functions of the antibodies is to stop microbes that are present at mucosal surfaces and in the blood from gaining access to and colonizing host cells and connective tissues. In this way Antibodies prevent infections from ever getting established. Antibodies do not have access to microbes that live and divide inside infected cells. Defense against such intracellular microbes is called cell-mediated immunity because cells called T-lymphocytes mediate it. Antibodies produced by B-lymphocytes are designed to specifically recognize extracellular microbial antigens, whereas the role of T-lymphocytes is to recognize antigens produced by intracellular microbes. Another important difference between B and $\mathrm{T}$ lymphocytes is that most $\mathrm{T}$ cells recognize only microbial protein antigens, whereas antibodies are able to recognize many different types of microbial molecules, including protein, carbohydrates, and lipids [5].

2) Properties of the Adaptive Immune Responses: The HIS is a very resilient mechanism with many desirable and advantageous properties. These properties are very diverse, hence somehow transferable to many different computational problems. The most important properties of the adaptive immune response include:

- Specificity: The ability to recognize and respond to many different microbes. This is illustrated by the observation that prior exposure to an antigen results in heighten responses to subsequent challenges with that antigen but not to challenges with other, even quite similar antigens. It is estimated that the immune system has the potential for distinguishing at least a billion different antigens or portions of antigens.

- Memory: Enhances responses to recurrent or persistent infections. Future infections will invoke the memory cells allowing for the immune system to mount larger and more effective responses. These secondary immune responses are usually more rapid, larger and better able to eliminate the antigen.

- Clonal Expansion: The ability to keep pace with rapidly proliferating microbes. The body can only maintain a certain number of immune cells. It does not keep an endless supply of immune cells that would not otherwise be used, wasting the body's resources. Only after an antibody binds the antigen, does it produce more antibodies of that specificity. Hence, HIS can adapt to a changing situation to utilize resources, as efficiently as possible.

- Specialization: Responses to distinct microbes are optimized for defense against the microbes. The antibody can recognize the patterns found on the receptors of antigen. If receptors for a certain antigen cannot be found, the HIS is able to produce receptors that match them through mutation or recombination of existing receptor combinations.

- Non-reactivity of self-antigens: This ability prevents injurious immune responses against host cells and tissues, by being able to distinguish between self and non-self.

- Autonomy and decentralized (distributed) control: The ability to function without outside interference or the need for a central controller. HIS can solve problems such as maintenance of cell population, repairing/replacing damaged cells when required and eliminating pathogens.

\section{Case Study: the Heating System Control}

\section{A. AIS Model}

Michael Mozer [6] undertook the task of controlling the heating system of an intelligent home system using AIS. This was inspired his an earlier work that addressed multiple aspects of the intelligent home control (iHome) using ANN. In Mozer's experiment, a network based AIS was develop to adapt to varying requirements and to learn on top of the usual control functions required. Requests to the home would be monitored and used as training data. Through this training the system was able to learn the typical user behaviour, but would still remain flexible to adapt to changes in usage. Lehmann and Dilger's [7] adapted the solution originally proposed by Mozer [6]. The approach involved monitoring user requests (antigens) and sending responses (antibodies) back to the system. Antibodies were produced according to the bone marrow model and a version of reinforcement learning mechanism. The architecture of the AIS for the heating system consists of three main components: the central control unit, the bone marrow and the AIS-network (Fig. 3). The central control unit is an interface to the external subsystems. Accordingly, the Central Control receives signals and generates from these signals the antigens, whilst in the opposite direction it transforms the network outputs into commands to the heating system. The bone marrow unit generates new B-cells and, if necessary, adapts them to the needs of the AIS-network. The AIS-network subsystem when stimulated produces antigens and antibodies using the operations of selection and mutation that are based on the affinity between the elements. 


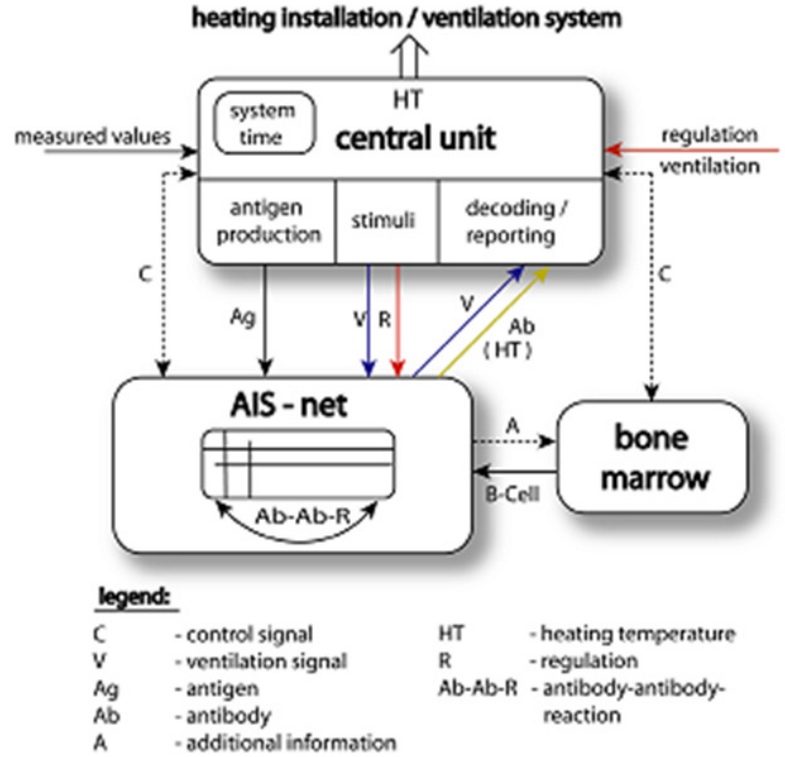

Fig. 3. The architecture of Lehmann and Dilger's AIS [7].

Lehmann and Dilger [7] examined scenarios specific to single rooms in the house, certain daytimes, and the weekdays. In any case, the intelligent home control system has to take into account the weather conditions outside. The most important factors that were regarded for the control of the heating system were the outdoor temperature, indoor temperature, type of the room, frequency of use, daytime, weekday as well as ventilation. The results of Lehmann and Dilger's AIS system looked very promising as the solution was able to adapt quickly to spontaneous signals sent from the users and to readapt to the normal behaviour later. Various scenarios explored regular use with a break of two weeks of no use, regular use with a change of use after a number of weeks, irregular use with frequent changes, and the control that involved several rooms. In reported cases, the system adapted to the changing requirements well. With its simple concepts, the Lehmann and Dilger's model of the AIS-based system is transferable to other control adaptations like the herein proposed Smart Air Conditioning System.

\section{SACS SOLUTION}

\section{A. Overview of the Architecture}

The Smart Air Conditioning System (SACS) consists of four main components. These four main components include Network Manager, Bone Marrow and AIS-Network and the system data storage (Fig. 4). This section describes each of the SACS components in the context of chosen immune principles. The SACS architecture is similar to the model proposed by Lehmann and Wagner; however, it is somewhat simplified for the purpose of this work. Most noticeable changes relate to the Central Unit which is replaced with the Network Manger. In SACS, AIS-Network issues control signals to the Bone Marrow unit from the Network Manager. The system data storage component is included to manage data persistency. The system has been designed with distribution and extensibility in mind. Multiple instances of the Network Manager can be produced over a network to share the load or produce multiple results for antigens to get the best possible solution.

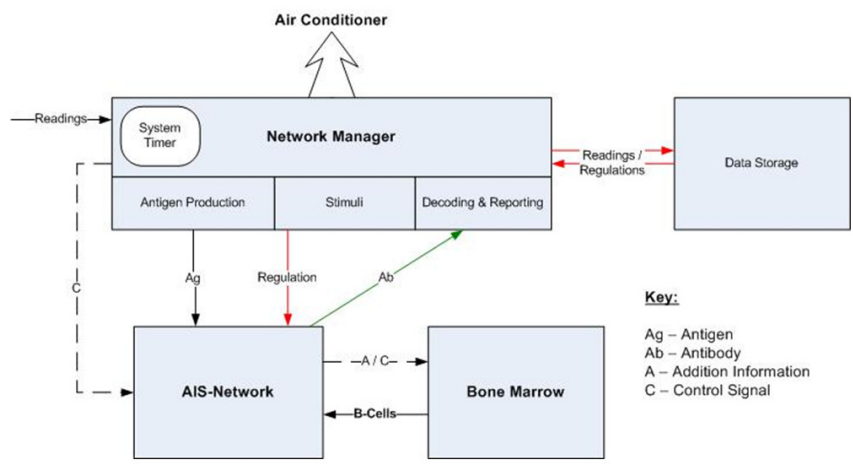

Fig. 4. Architecture of the system.

\section{B. Network Manager}

The Network Manager is the interface between the AIS and the outside world. It also interfaces with the system data store saving and retrieving configuration and environment information, measurements from the hardware and regulation commands from the users (Fig. 5). With respect to the AIS, when the system timer elapses the Network Manager starts producing antigens from the measured values and stimuli from regulation commands and then it initiates the update.

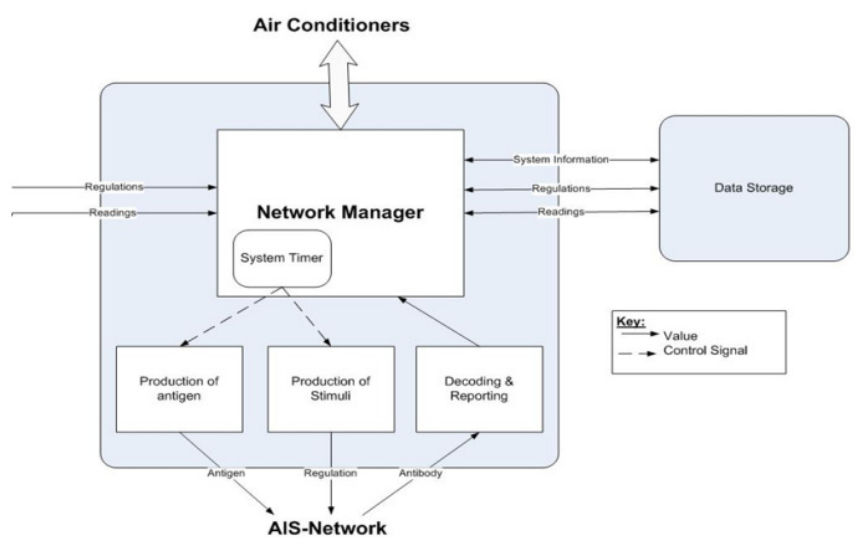

Fig. 5. Structure of network manager.

The Network Manager also decodes the information delivered by the antibodies. In addition, it controls the AIS-Network through the use of a number of parameters. Among them are the initial size of the population, the size of the whole network, the number of best suited elements to select, the number worst suit elements to remove, mutation rate controller, suppression and pruning thresholds.

\section{The AIS Network}

If the Network Manager is "the brains" of the SACS, then the AIS-Network is its "heart". Through cooperation with the network manager and the bone marrow, it determines 


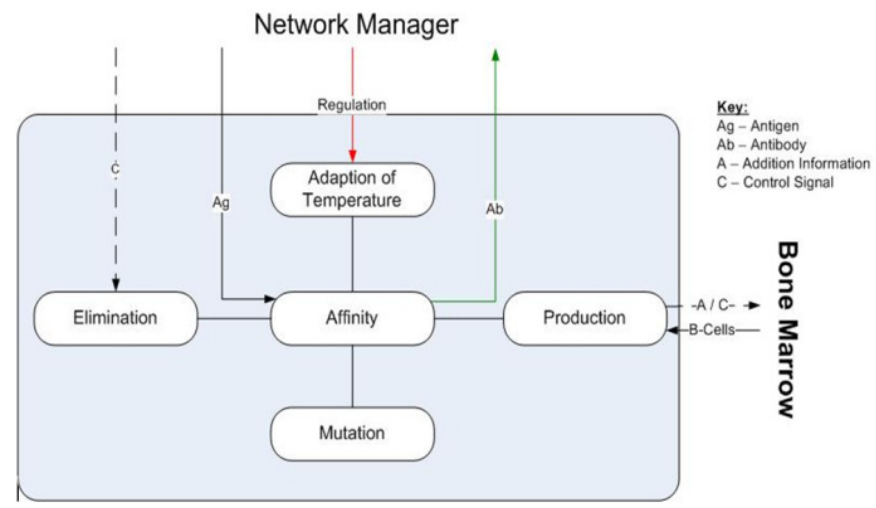

Fig. 6. Structure of AIS network.

the antibodies that best match the supplied antigens. During the process of finding the best matching antibodies, the AISNetwork will ask the Bone Marrow to generate more B-cells, matching antibodies will be cloned and the clones that are below the threshold will be eliminated. It should be noted that the Network Manager periodically tells the AIS-Network to eliminate B-cells that are considered too old to be of any value. One of the key methods of the aiNet Class is Update(). Its procedure is somewhat similar to Lehmann and Dilger's, however, it was significantly altered to suit the needs of SACS solution. The samples immune-computing algorithms are presented in Fig. 7 and Fig 8.

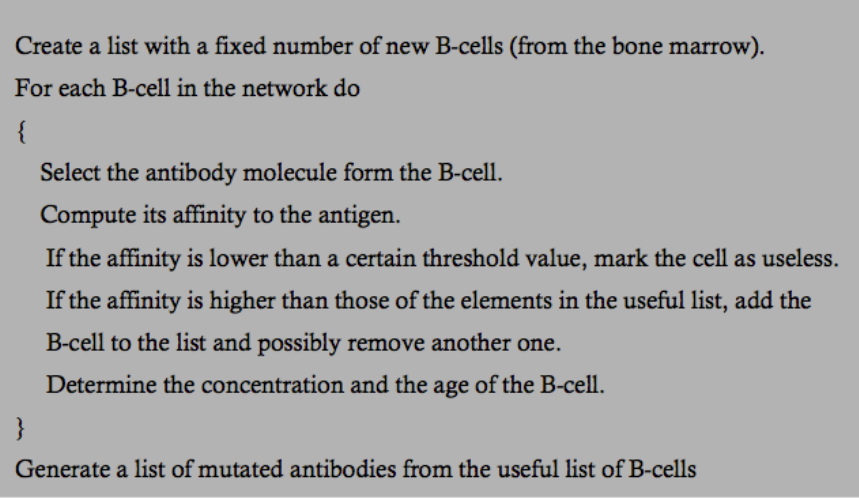

Fig. 7. AIS network

Determine the amount of clones and the mutation rate.

Generate the mutated antibodies according to the mutation rate

Store the antibodies in a special list.

Eliminate antibodies whose affinity is lower than that of the last element in the list of best B-cells.

Eliminate a predefined number of elements from the list of useless elements.

Add the clones to the network from the special list.

Before returning the best of the B-cells for the current zone, adapt it according to the regulation.

Fig. 8. AIS network

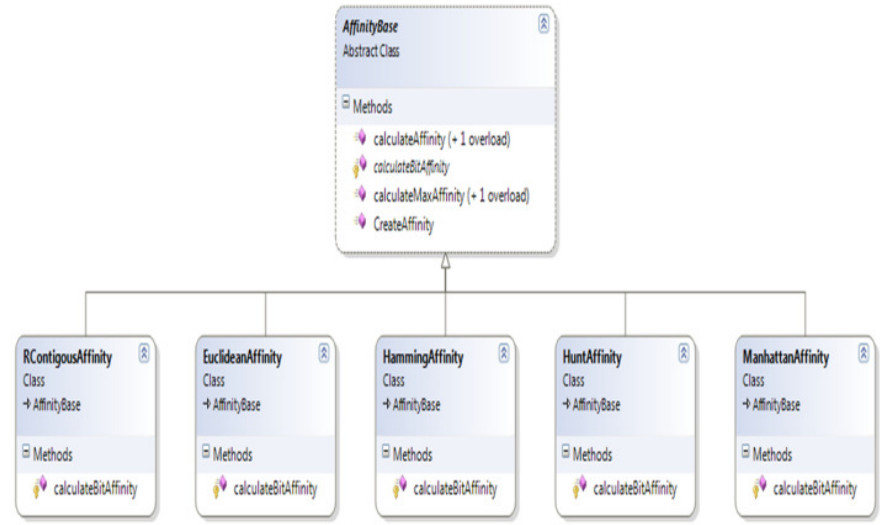

Fig. 9. Affinity class diagram.

The polymorphism and inheritance principles are designed into the system (Fig. 9). The abstract class AffinityBase has an overridable method called calculateBitAffinity() that determines the affinity value when comparing 2-bit strings. Should a new method for calculating the affinity be required one needs simply to add a new class that inherits from AffinityBase that implements the required calculation in calculateBitAffinity(). Similarly, if an alternative mutation algorithm is required, the class Mutator can be extended by overriding the mutate() method.

The database is implemented in SQLite, a self-contained, serverless, zero-configuration, transactional SQL database engine that is commonly embedded in many other systems. Object Relational Model (Fig. 10) is used to access the tables rather than SQL. This allows a better use of OO principles and mappings between the objects and tables.

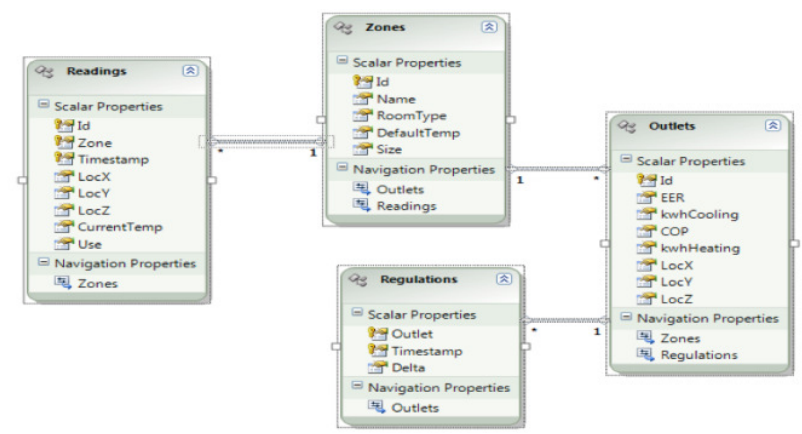

Fig. 10. Relational model.

\section{EXPERIMENTATION AND ANALYSIS}

\section{A. Affinity Comparison Tests}

The affinity matching is crucial for AIS algorithms, as the selection of a wrong affinity can lead to ineffective detection. Several experiments using minimal variance scenarios in user patterns were run to compare various affinity matching measures and test which perform better in different circumstances. Although, the Hamming distance is not as linear as Euclidean distance and not as exponential as Hunt distance, for most experiments, the Hamming distance was used as the affinity 
measure in all tests it consistently performed well for all its variation between the lowest and highest values. Findings for the first set of tests mostly conformed to what was expected. Some interesting behaviours where observed with various affinity measure methods. The test that involved changing the Zone variable resulted in undesirable bias using all the affinity methods. This could be attributed to automatically generated id values used in the comparisons, resulting in a bias towards the zone when it was added to the system. Similarly, changing the Time-of-the day or Day of the Week variables showed a bias towards time/days in the week that were closer to the antigen value. Lastly, changes to Frequency of use exhibited the expected behaviour with a bias away from None. Fig. 11 shows how affinity measures performed with the antigen frequency of use set to one of the possible values from low (top-left) to high (bottom-right).

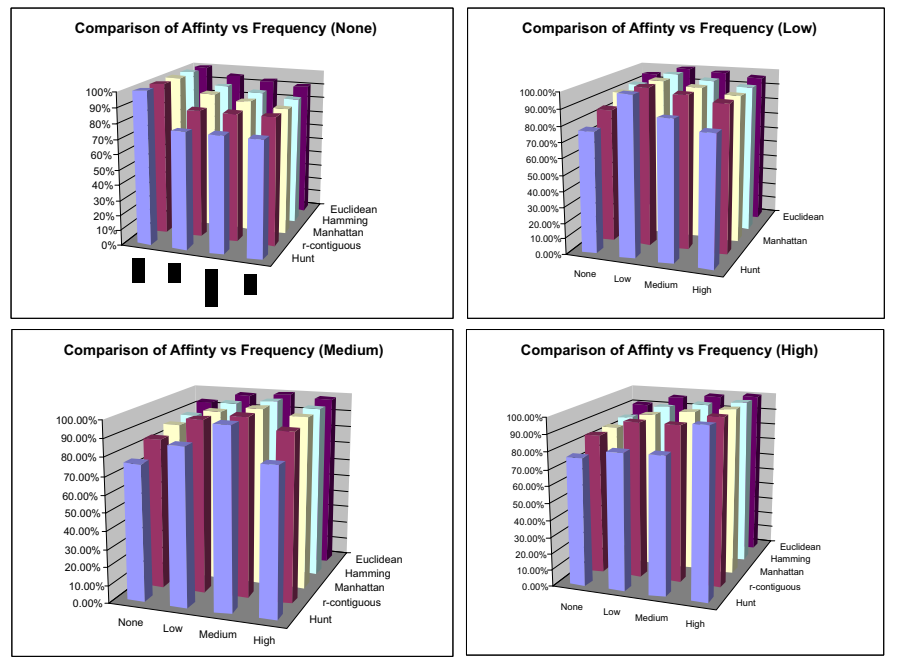

Fig. 11. Comparison of affinity vs. frequency of use.

\section{B. User Pattern Variance Tests}

These tests investigated the effectiveness of the AIS algorithm in detecting and adapting to the user's needs. The aim was to see how fast the algorithm returns to normal behaviour once the variance has stopped. For these test, a dedicated GUI was designed (Fig. 12) to allow monitoring the system usage for prolonged period of time. The findings for the user pattern variance tests showed the algorithms behaved as expected although converging faster than anticipated.

For example, the default temperature for a selected zone was $27^{\circ} \mathrm{C}$ while the sensor reading was $23^{\circ} \mathrm{C}$. There were no regulations from the defaults and the system operated at a constant except for early hours (before 8 a.m.). Other tests included variations in a predefined period of time. In most cases, the system behaved well adapting to the changes. For simulations that run for several days, the final days sometimes did not show any change to the optimal temperature. The early convergence of the AIS with little changes raises questions on the global variable values, in particular mutation control and clone multiplier. By raising the mutation control variable the system should increase the chances of antibody mutation

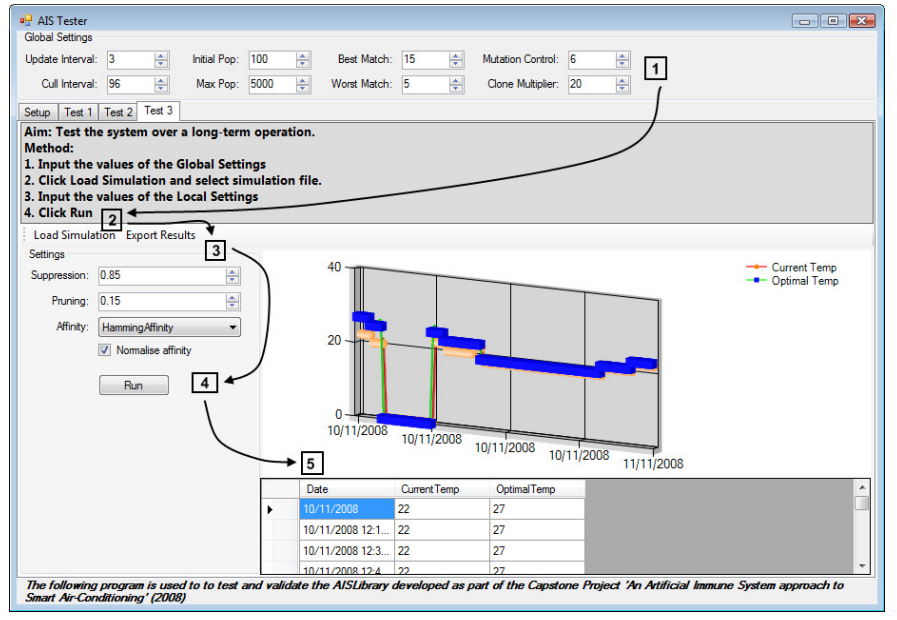

Fig. 12. Inputs and outputs GUI showing a user pattern variance test.

to allow for greater variance in the network. The amount of clones produced may also be too little to reduce the number of new mutated antibodies added to the system. Lastly, the suppression threshold may be too high and excluding too many B-cells from the system. The accuracy of the system was less than expected which implies that the some variables required further adjustments to find a better balance between how fast the system can learn and how accurate it is in matching.

\section{CONCLUSION}

The development of AIS did not come without its fair share of problems. By overcoming these problems, AIS is able to demonstrate its full potential as an optimisation heuristics. The effectiveness of these computing paradigms cannot be measured to their entirety. However, by looking at the example of genetic algorithms, it is possible to visually encompass the simplicity and versatility of the algorithms. The main argument is not 'if' AIS models are able to compete against other optimisation and search problem heuristics, but rather appreciate their ability to integrate with other natural computing paradigms. Research of these branches of AIS is propelling its development and is something to watch in the future. As AIS is coming of age, there is much more understanding and knowledge and cooperation amongst scholars.

\section{REFERENCES}

[1] D. R. Barr, G. K. Poock, and F. R. Richards, Experimentation Manual Part 1: Experimentation Methodology. Monterey, California: Naval Postgraduate School, 1978.

[2] C. Charras and T. Lecroq, "Exact string matching algorithms," 1997, retrieved 13/02/12 from http://www-igm.univ-mlv.fr/ lecroq/string/.

[3] C. A. C. Coello, D. C. Rivera, and N. C. Cortes, "Use of an artificial immune system for job scheduling," in Artificial Immune System, J. Timmis, P. Bently, and E. Hart, Eds. Berlin: Springer-Verlag, 2003 pp. $1-10$.

[4] D. Dasgupta, "Advances in artificial immune systems," IEEE Computational Intelligence Magazine, vol. 1, no. 4, pp. 40-49, 2006.

[5] L. N. de Castro and F. J. von Zuben, "aiNET: An artificial immune network for data analysis," in Data Mining: A heuristic approach, H. A. Abbass, R. A. Sarker, and C. Newton, Eds. Idea Group Publishing, 2001, pp. 231-259.

[6] M. C. Mozer, "12 lessons from an adaptive house," in Smart environments: Technologies, protocols, and applications, D. Cook and R. Das, Eds. J. Wiley \& Sons, 2005, pp. 273-294. 
[7] M. Lehmann and W. Dilger, "Controlling the heating system of an intelligent home with an artificial immune system," in International Conference on Artificial Immune Systems, H. Bersini and J. Carneiro, Eds. Berlin: Springer-Verlag, 2006, pp. 335-348.

[8] E. Bendiab, "Artificial immune system for multimodality image alignment," in Artificial Immune System, J. Timmis, P. Bently, and E. Hart, Eds. Berlin: Springer-Verlag, 2003, pp. 11-19.

[9] L. N. de Castro and J. Timmis, Artificial Immune Systems: A Computational Intelligence Approach. London: Springer-Verlag, 2002.

[10] L. N. de Castro and F. J. von Zuben, Recent Developments in Biological Inspired Computing. Hershey: Idea Group Publishing, 2005.
[11] D. Dumitrescu, B. Lazzerini, L. Jain, and A. Dumitrescu, Evolutionary Computation. USA: CRC Press, 2000.

[12] D. Graupe, Principles of Artificial Neural Networks. Singapore: Word Scientific Publishing, 1997.

[13] C. A. Janeway Jr, P. Travers, M. Walport, and M. Shlomchik, Immunobiology: the Immune System in Health and Disease, 5th ed. New York: Garland Publishing, 2001.

[14] M. Middlemiss, Framework for intrusion detection inspired by the immune system. University of Otago, 2005, Discussion Paper. 